5 Research Square

\title{
The effect of spiritual counseling on improving of the quality of life among pregnant women: a randomized control trials
}

Masomeh MonfaredKashki

Zanjan University of Medical Sciences

Azam Maleki ( $\square$ malekia@zums.ac.ir)

Zanjan University of Medical Sciences

Kourosh Amini

Zanjan University of Medical Sciences

\section{Research Article}

Keywords: Quality of life, pregnancy, spirituality, women's health, psychological factor, childbirth

Posted Date: December 15th, 2020

DOI: https://doi.org/10.21203/rs.3.rs-110055/v1

License: (1) (i) This work is licensed under a Creative Commons Attribution 4.0 International License.

Read Full License 


\section{Abstract}

Background: To examine the effect of spiritual counseling on the quality of life (QoL) of women with the first time pregnancy.

Methods: This randomized control trial was carried out on 60 pregnant women who recruited at two childbirth preparation centers of Zanjan (Iran) in 2018. The eligible women were allocated into two intervention and control groups according to the 4-block design. The spiritual counseling was carried out in eight sessions, two times per week at 20 -32 weeks of gestation. The control group only received routine cares. The quality of life were measured before and two months after intervention.

Results: Overall in pre intervention phase, the quality of life did not show a statistically significant difference between the two groups ( $p>0.05)$. In Post the intervention, the overall QoL showed a statistically significant difference between the two groups $(p<0.05)$. But the physical functioning, body pain, and social functioning domains were not statistically significant $(p>0.05)$. According to the linear regression, the risk of poor quality of life in the intervention group was 0.78 times less than of the control group $(p=0.0001)$.

Conclusion: Spiritual counseling had a positive impact on improving of QoL in the first time pregnant women. The integration of spiritual counseling in the childbirth education package could be improved the psychological aspect of quality of life more than the physical aspect of quality of life. It can be considered used by healthcare providers for planning childbirth interventions.

Trial registration: The study was registered at the Iranian Registry of Clinical Trials under the number IRCT20150731023423N12, 2018-11-06

\section{Project Summary}


Title The effect of spiritual counseling on improving of the quality of life among pregnant women: A randomized control trials

Background Physical and psychological changes during pregnancy affect the social and physical performance, as well as the quality of life (QoL) of pregnant women. There is a gap in the effectiveness of spiritual-based interventions in the culture and context of Iran on health-related QoL in women with the first pregnancy

Methodology Parallel Randomized control trial- Not blinded

Intervention: Spiritual counseling was held in 8 sessions+ routine care

Control: routine care

Study $\quad$ Expected recruitment start date $=2018-11-11$

Duration

Expected recruitment end date $=2019-02-22$

Study centers

Two childbirth preparation centers in Zanjan city, Iran

Objectives To examine the effect of spiritual counseling on the quality of life (QoL) of women with the first time pregnancy

Number of $\quad 60$ randomized patients in two arms; Treatment and Control

Subjects

Main Inclusion Criteria

Inclusion

Criteria

Living in Zanjan city,Gestational age 20-24 weeks, willingness to participate in the study, lack of any psychiatric disorders or use of psychiatric drugs, the Edinburgh Depression Inventory Score $\leq 10$ \& the Cohen Perceived Stress 18 to 36 ,having a normal pregnancy with a singleton fetus

\section{Exclusion criteria}

Having any medical or obstetric complication, being absent more than two counseling sessions, no access to telephone

Outcome Quality of life among pregnant women

Measure: Health related Quality of life(SF-36)

\section{General Information}


Protocol title

protocol identifying number

Name and address of the sponsor/funder.

Name and title of the investigator(s) who is (are) responsible for conducting the research, and the address and telephone number(s) of the research site(s), including responsibilities of each.
The Effectiveness of Spirituality Counseling on Quality of Life in First Time Pregnant Women

32031

Dr Alireza Shoghli

Address: Azadi Blvd, Zanjan University Of Medical Sciences, Zanjan. Postcode No: 4515613191, Tel: +98 2433148300

Maleki Azam, PhD in Maternal \& Child Health

Address 1: Gavazang Road, Zanjan University Of Medical Sciences, Zanjan, Iran, Tel: +98 24334206519

Address2: Azadi Square ,Jomhori Eslami St, Social Determinants of Health Research Center, Zanjan University of Medical Sciences, Zanjan, Iran, Phone: 024-33156141

Fax: 024 - 33424770, Postal code: 4515613191, Email: malekia@zums.ac.ir\&malekia41@yahoo.com. ORCID : https://orcid.org/0000-0001-7888-1985

Social Determinants of Health Research Center, Zanjan University of Medical Sciences, Zanjan, Iran.

Name of recruitment center:

Childbirth Preparing Class at Imam Hussein Hospital \& Ayatollah Mousavi Hospital of Zanjan, Iran

All participants in the study (intervention and control) received routine care, and the intervention, which was in the form of training and counseling, was integrated with the content of the routine care. It was held only the intervention group. The educational intervention did not have any harm to the participants and it was always possible to cancel the study for all participants.

The counseling was conducted by a midwife that familiar with counseling approaches under the supervision of a clinical psychologist. A standard questionnaire was used for data collection.

\section{Introduction}

Pregnancy is a physiological phenomenon in the women's life. Physical and psychological changes during pregnancy can affect the social and physical performance, as well as the quality of life (QoL) of pregnant women (1). The quality of life (QoL) reflects the subjective perceptions of the individual's situation in life based on the cultural and value system, given the individual's goals, expectations, 
standards and attitudes(2). According to the World Health Organization (WHO), health-related QoL refers to the physical, psychological, social, and spiritual dimensions of individuals' well-being (2). Furthermore, QoL of pregnant women could be affected by many factors such as gestational age, the social and economic support, and complications before or during pregnancy $(3,4)$. On the other hand, poor pregnancy QoL is associated with adverse outcomes for example preterm labor pain, and pregnancy related symptoms such as fatigue, low back and pelvic pain $(5,6)$ Additionally, low QoL in pregnancy contributes to low QoL in the postnatal period (7).

Spirituality and religiosity are known as an important components of health and well-being(8). Accordingly, the European Commission recommends that spiritual care be provided to nursing and midwifery care, to meet the spiritual, religious and cultural needs of the people(9).

Childbearing is one of the ideal condition for enriching spirituality. Some people believe that the process of pregnancy and childbirth as a time to get closer to God and make life more meaningful(10).

Spirituality is defined as sensitivity or attachment to religious values, or to things of the spirit as opposed to material or worldly interests(11). Spiritual experience is a unique experience and includes understanding the meaning of life, positive life experience, feeling happy and life satisfaction(12).

In Iran, spiritual care has not routinely included in prenatal care program, while in recent years, valuable results from the implementation of interventions based on religion and spirituality in improving anxiety, depression and coping with stress has been reported $(13,14)$. The use of spiritual counseling alone or in combination with cognitive-behavioral therapy can help with improving QoL in women with high risk pregnancy, postpartum depression, and fear of labor pain (15-18). However, there is a gap in the effectiveness of spiritual-based interventions in the culture and context of Iran on health-related QoL in women with the first pregnancy. Given the importance of spiritual care and the presence of limited studies in this field, this study aimed to determine the effect of spiritual counseling on improving of the QoL of women with the first time pregnancy.

\section{Method}

\section{Study design and setting}

This parallel randomized control trial was carried out on 60 first time pregnant women who referred to two childbirth preparation centers in Zanjan city, Iran in 2018-19.

\section{Aims}

To examine the effect of spiritual counseling on the quality of life (QoL) of women with the first time pregnancy.

\section{Participant}


Given the indicator of a similar study, with considering , power of $80 \%$, and attrition rate of $15 \%$ (17), the sample size of the present study was calculated 30 first time pregnant women in each group.

Inclusion criteria consisted of living in Zanjan city, gestational age of 20-24 weeks, willingness to participate in the study, lack of any psychiatric disorders or use of psychiatric drugs, obtaining scores $\leq 10$ according to Edinburgh Postnatal Depression Scale (EPDS), scores 18 to 36 based on the Cohen Perceived Stress Scale (PSS), and having a normal pregnancy with a singleton fetus.

Exclusion criteria were the presence of any medical or obstetric complication during the study period, being absent more than two counseling sessions, and no access to telephone follow up.

\section{Procedure\& randomization}

Pregnant women who met the inclusion criteria and signed the informed consent form were allocated into two intervention and control groups using randomized a block size of four. To ensure the concealment of the sequence of enrolment, an opaque sealed envelope system was used (19). Envelope preparation and random allocation sequencing were performed by a person not involved in the research process. In the present study, participants \& researcher were not blinded only outcome assessors were blinded. The research process is showen in fig1.

\section{Intervention}

The counseling sessions were designed in accordance with the study by khoda Karmari et al. (20) and the method suggested by Richard and Bergin's (21).

According to the guidelines of the Iranian Ministry of Health, routine childbirth preparation classes were held from the 20th week of gestation every two weeks until the 32nd week of gestation. The sessions focused on making the mothers familiar with the different stages of pregnancy from fertilization to delivery, personal hygiene, nutrition, mental and physical changes during pregnancy, pregnancy risks, childbirth planning, postpartum health, breastfeeding, and child care. However, no spiritual counseling was provided.

The control group only received routine care. A spiritual counseling were integrated with routine care in the intervention group. Spiritual counseling was held in 8 sessions, as a group counseling (8-10 people) for 4 weeks ( 2 sessions per week for 45 minutes) at preparation classrooms. The main topic of counseling were reported in table 1.

Each session was started with a focus on breathing exercise or the sacred name like "Allah". Next, the counselor provided a description of the subject of the meeting and encouraged the mothers to express emotions, needs, concerns and thoughts on pregnancy. At the same time, the counselor guided the participants to increase their knowledge to choose the appropriate remedy for emotional reactions during pregnancy and pay attention to spiritual aspects of life. Further advice was given as homework. At the end of each session, explanations and summaries was provided and the women discussed about the 
topic. The counseling was conducted by a midwife that familiar with counseling approaches under the supervision of a clinical psychologist.

\section{Outcomes}

The outcome of this study was prenatal QoL of the first time pregnant women. Data were collected using the SF-36 as a standard questionnaire of QoL, which was completed by the groups before and two months after the intervention.

\section{Data collection instruments}

\section{Demographic}

It included personal information of woman's age, education, occupation and spouse's occupational status.

Health-related quality of life (HRQoL) -SF-36

It is a health-related QoL (HRQoL) questionnaire as a multidimensional measure of health status for self or interviewer administration. It is widely used in clinical research and is a reliable and valid measure of health-related QoL in different populations $(22,23)$. It measures the perceptions of health-related QoL in 8 domains of Physical Functioning, Role-Physical, Bodily Pain, General Health, Vitality, Role-Emotional, Social Functioning and Mental Health. Responses are scored on a 5-point scale, that are transformed into a score of $0-100$ with higher scores indicating better functioning or well-being. Validity and reliability of The Farsi version of the questionnaire have been assessed by Montazeri et al. (23).

\section{Data analysis}

The statistical analysis was performed using the SPSS software version 16. Descriptive statistics were employed to describe demographic data. The chi-square test was used to compare the demographic characteristics between the groups. The Kolmogorov-Smirnov test revealed that the scores of the QoL and its components had normal distributions. Therefore, to compare total scores and all areas between and within the groups in pre- and post-intervention, the independent t-test and paired samples t-test were applied, respectively. The risk of having a low QoL was estimated using the linear regression model. The level of significance was $p<0.05$.

\section{Results}

The Participant flow is showen in fig 1.

There were not statistically significant differences between the two groups before the intervention in terms of demographic characteristics. So that, the groups were homogeneous. Also, the mean age of the participants showed that there was no significant difference between the two groups and they were homogeneous in terms of this variable $(p<0.05)$. (Table 2$)$ 
The mean score of total QoL and all its domains were greater in the control group compared to intervention group and the differences between the groups were not statistically significant therefore the two groups are homogeneous before the study.

The post test showed that the overall score of QoL and the area of "Role-Physical, General Health, Vitality, Role-Emotional, and Mental Health" were greater in the intervention group compared to the control group, and the difference between the two groups were statistically significant. While in term of "Physical Functioning, Bodily Pain, and Social Functioning" domains, no statistically significant differences were reported $(p>0.05)$.

Comparing before and after scores of QoL and its domains in the control group showed no statistically significant differences $(p>0.05)$.

Comparing before and after scores of QoL and the area of "Physical Functioning, Role-Physical, General Health, Vitality, Role-Emotional, and Mental Health" in the intervention group showed statistically significant improvements $(p<0.05)$. Only the scores of two domains of "Bodily Pain, Social Functioning "were not statistically significant ( $p>0.05)$ (Table3).

According to the results of linear regression, the risk of poor QoL in the intervention group was 0.78 times less than that of the control group $(p=0.0001)$ (table 4$)$.

\section{Discussion}

The study results showed that spiritual-based counseling could be improved the overall QoL. However, three domains of QoL (physical function, bodily pain, and social function) showed no improvements. The current study emphasized that the spiritual counseling approach improved the psychological aspects of QoL more than the physical aspects. Limited information is available on the effectiveness of spiritual counseling for improving the QoL of the first time pregnant women. However, our results were consistent with some studies that conducted on multiparous or high-risk pregnancy samples (15-18). Our results were also consistent with the studies that used different procedures for intervention, for example, Bashirpour et al (2018) showed that the mindfulness-based approach (24), Liu et al (2019) suggested that yoga or physical activity could be improved the QoL and well-being of pregnant women(25). O'Connor et al. (2018) also reported similar results (26). Our study results emphasized that the spiritual counseling approach compared to other methods can be equally improved the QoL of pregnant women.

Various ideas have been reported concerning the biological and psychological effects of spiritual experience on diseases have been emphasized in some studies. It can be claimed that some cognitive patterns, psychological characteristics and behavioral patterns created by spirituality-oriented methods lead to strengthening health and improving the physiological function of the body and consequently increase the psychological resistance of the person in poor physical and social situations. Accordingly, religious and spiritual practices lead to increased tolerance, patience, self-control, satisfaction, emotional control, optimism, self-efficacy (based on trust in God's blessing), altruism, kindness and love $(12,27,28)$. 
Religious rituals and spirituality can increase QoL through changing people's attitudes, increasing their sense of responsibility towards themselves and others, promoting the search for meaning in the life, having a greater sense of happiness and self-esteem(29).

The effectiveness of the spiritual approach on improving QoL in the different population (30-32) has been shown that spirituality is a universal element (33). Belief in God creates a change in the perspective toward the life(21). The spiritual aspects of pregnancy and childbearing are often neglected in the literature. Integration of midwifery led counseling with the spiritual approach for improving quality of life of women is necessary.

As a limitation, data collection was performed using a self-report questionnaire. Sampling was conducted on the participants of childbirth preparation classes, which can affect the generalizability of findings. Also, the long duration of each session could lead to the exhaustion of mothers. However, the women were allowed to have rest and walk for a few minutes during the sessions. Finally, the short follow-up period should be considered by researchers in future studies. The spouses' of women not included in this study that should be considered in future studies.

\section{Conclusion}

This revealed that the spiritual counseling had a positive impact on improving the QoL of first time pregnant women. The integration of spiritual counseling with the educational content of childbirth preparation can improve the psychological aspect of QoL of pregnant women more than the physical aspect. Therefore, it can be used for planning suitable interventions among pregnant women.

\section{Key Points}

Poor QoL in pregnancy is associated with adverse pregnancy outcomes

The spiritual counselling had a positive impact on improving the QoL of first time pregnant women.

Integration of midwifery led counselling with the spiritual approach for improving childbirth preparation content is necessary.

\section{List Of Abbreviations}

$\mathrm{HQoL}=$ Health-related quality of life

QoL= Quality of life

Edinburgh Postnatal Depression Scale (EPDS)

Perceived Stress Scale (PSS) 


\section{Declarations}

Ethical Approval: This article was a part of MSc thesis and approved by the Ethics Committee of the Vice Chancellor for Research of Zanjan University of Medical Sciences, Iran, with the approval number IR.ZUMS.REC.1397.024. All procedures of the study were in accordance to the protocol of the regional ethical research committee and with the declaration of Helsinki 1964. After informing the study's purposes, written consent were obtained from all women. They were informed that their participation were voluntary, confidential, and anonymous, and were apprised of their right to withdraw from the research at any time. The study was registered at the Iranian Registry of Clinical Trials under the number IRCT20150731023423N12, 2018-11-06

\section{Consent for publication}

Not applicable.

\section{Availability of data and materials}

The data sets used and analyzed during the current study are available from the corresponding author on reasonable request

\section{Competing interests}

No potential conflict of interest relevant to this article was reported

Funding Sources. This article was part of MSc thesis and funded by the Research Deputy of Zanjan University of Medical Sciences, Iran, with the approval number (The code " A-11-344-8").

\section{Acknowledgements}

We would like to thank the Zanjan University's vice-chancellor of education and vice-chancellor of research and technology for their financial support to carry out the study (The code " A-11-344-8"). We would like to thank the staffs for their collaboration, and mothers for their participation

\section{Authorship}

This study was one part of the MSC thesis of M.K,M. The conception, design of the study, and data collection process were undertaken by M.K,M and A.M was the supervisor who also contributed to the design of the study and reporting of the results. K.A and S.F as the second supervisors contributed to all the stages of the study. Analysis, interpretation, and reporting were supervised by S.F and A.M. All authors contributed to the drafting and revising of the article and are in agreement with final version of the manuscript to be submitted to the journal; they also meet the criteria of authorship. S.F was one of the colleagues, but unfortunately he is not alive.

\section{References}


1. Lau Y, Yin L. Maternal, obstetric variables, perceived stress and health-related quality of life among pregnant women in Macao, China. Midwifery. 2011;27(5):668-73.

2. Group TW. The World Health Organization quality of life assessment (WHOQOL): development and general psychometric properties. Social science \& medicine. 1998;46(12):1569-85.

3. Calou CGP, Pinheiro AKB, Castro RCMB, de Oliveira MF, de Souza Aquino P, Antezana FJ. Health related quality of life of pregnant women and associated factors: An integrative review. Health. 2014;6(18):2375.

4. Lagadec N, Steinecker M, Kapassi A, Magnier AM, Chastang J, Robert S, et al. Factors influencing the quality of life of pregnant women: a systematic review. BMC pregnancy and childbirth. 2018;18(1):455.

5. Wang P, Liou S-R, Cheng C-Y. Prediction of maternal quality of life on preterm birth and low birthweight: a longitudinal study. BMC pregnancy and childbirth. 2013;13(1):124.

6. Lau Y. The effect of maternal stress and health-related quality of life on birth outcomes among Macao Chinese pregnant women. The Journal of perinatal \& neonatal nursing. 2013;27(1):14-24.

7. Fobelets M, Beeckman K, Buyl R, Daly D, Sinclair M, Healy P, et al. Mode of birth and postnatal healthrelated quality of life after one previous cesarean in three European countries. Birth. 2018;45(2):13747.

8. Puchalski CM, editor The role of spirituality in health care. Baylor University Medical Center Proceedings; 2001: Taylor \& Francis.

9. McSherry W, Ross L, Attard J, van Leeuwen R, Giske T, Kleiven T, et al. Preparing undergraduate nurses and midwives for spiritual care: Some developments in European education over the last decade. Journal for the Study of Spirituality. 2020;10(1):55-71.

10. Crowther S, Stephen A, Hall J. Association of psychosocial-spiritual experiences around childbirth and subsequent perinatal mental health outcomes: an integrated review. Journal of Reproductive and Infant Psychology. 2020;38(1):60-85.

11. https://www.ncbi.nlm.nih.gov/mesh/?term=spirituality.

12. Srivastava A, Krishna A. Psycho-Physiological Mechanism of Spirituality-Health Relationship: Theoretical Conceptualisation. J Indian Health Psychol.2(1):1-9.

13. Jabbari B, Mirghafourvand M, Sehhatie F, Mohammad-Alizadeh-Charandabi S. The effect of holly Quran voice with and without translation on stress, anxiety and depression during pregnancy: a randomized controlled trial. Journal of religion and health. 2020;59(1):544-54.

14. Kamali Z, Tafazoli M, Ebrahimi M, Hosseini M, Saki A, Fayyazi-Bordbar MR, et al. Effect of spiritual care education on postpartum stress disorder in women with preeclampsia. Journal of education and health promotion. 2018;7.

15. Moazedi K, Porzoor P, Pirani Z, Adl H, Ahmadi H. The Effectiveness of Islamic Teaching Based Religious-Spiritual Psychotherapy on Quality of Life, in Infertile Women. Journal of Health. 2019;9(5):589-98. 
16. NIAZ AM, Abdollahi M, ZABIHI HNK, Ashoori J. Effect of Spiritual Group Therapy on Anxiety and Quality of Life among Gestational Diabetic Females. 2017.

17. Zamani SN, Zarei E, Haji Alizadeh K, Naami AZ. Effectiveness of Combination of CognitiveBehavioral Therapy and Resilience Training Based on Islamic Spirituality and Cognitive Flexibility on Postpartum Depression, Fear of Labor Pain and Quality of Life. Hormozgan Medical Journal. 2018;22(4):e86489.

18. Hunter L, Bormann J, Belding W, Sobo EJ, Axman L, Reseter BK, et al. Satisfaction and use of a spiritually based mantram intervention for childbirth-related fears in couples. Applied Nursing Research. 2011;24(3):138-46.

19. Doig GS, Simpson F. Randomization and allocation concealment: a practical guide for researchers. Journal of critical care. 2005;20(2):187-91.

20. Khodakarami B, Bibalan FG, Soltani F, Soltanian A, Mohagheghi H. Prognostic role of spiritual intelligence components in pregnant women's depression, anxiety, and stress. Health, Spirituality and Medical Ethics. 2016;3(2):16-23.

21. Richards PS, Bergin AE. A spiritual strategy for counseling and psychotherapy. 1997.

22. Montazeri A, Goshtasebi A, Vahdaninia M, Gandek B. The Short Form Health Survey (SF-36): translation and validation study of the Iranian version. Quality of life research. 2005;14(3):875-82.

23. Lins L, Carvalho FM. SF-36 total score as a single measure of health-related quality of life: Scoping review. SAGE open medicine. 2016;4:2050312116671725.

24. Basharpoor S, Soleymani E, Kazemi N, Nabi Salehi M. THE EFFECTIVENESS OF MINDFULNESSBASED STRESS REDUCTION TRAINING ON IMPROVING THE QUALITY OF LIFE AND WELL-BEING OF PREGNANT WOMEN. The J Urmia Nurs Midwifery Fac. 2018;16(6):445-54.

25. Liu N, Wang J, Chen D-d, Sun W-j, Guo P-p, Zhang X-h, et al. Effects of exercise on pregnant women's quality of life: a systematic review. European Journal of Obstetrics \& Gynecology and Reproductive Biology. 2019.

26. O'Connor P, Poudevigne MS, Johnson KE, de Araujo Brito J, Ward-Ritacco CL. Effects of Resistance Training on Fatigue-Related Domains of Quality of Life and Mood During Pregnancy: A Randomized Trial in Pregnant Women With Increased Risk of Back Pain. Psychosomatic medicine. 2018;80(3):327-32.

27. Seybold KS, Hill PC. The role of religion and spirituality in mental and physical health. Current Directions in Psychological Science. 2001;10(1):21-4.

28. Abdollahpour S, Khosravi A. Relationship between spiritual intelligence with happiness and fear of childbirth in Iranian pregnant women. Iranian journal of nursing and midwifery research. 2018;23(1):45.

29. Lent RW. Toward a unifying theoretical and practical perspective on well-being and psychosocial adjustment. Journal of Counseling Psychology. 2004;51(4):482.

30. HAMID N, KHODADOST F, MEHRABIZADEH HM. PREDICTION OF LIFE QUALITY AND DAILY ACTIVITIES OF KIDNEY TRANSPLANTPATIENTS IN AHVAZ ACCORDING TO SPIRITUAL 
INTELLIGENCE, HEALTH CONTROLSOURCE AND COPING STRATEGIES. 2017.

31. Manshaee G, Haji Mohammad Kazemi S, Ghamarani A. Prediction Model of Quality of Life Promotion on the Basis of Emotion Expression, Spiritual Intelligence and Pain acceptance in Female Patients with Fibromyalgia. Anesthesiology and Pain. 2018;8(4):60-73.

32. Dalmida SG. Spirituality, mental health, physical health, and health-related quality of life among women with HIV/AIDS: integrating spirituality into mental health care. Issues in mental health nursing. 2006;27(2):185-98.

33. O. Harrison M, Koenig HG, Hays JC, Eme-Akwari AG, Pargament KI. The epidemiology of religious coping: A review of recent literature. International review of psychiatry. 2001;13(2):86-93.

\section{Tables}

Table 1 Details of the intervention 


\section{Counselling content}

The first session was to meet the participants and researcher, to explain the aim, the rules, and brief full program, providing pre-test.

Talking about concept of quality of life, self-concept in pregnancy, and checking misconceptions.

Assessing attitudes and beliefs of the pregnant women on spiritual issues, the role of god and religious in her life.

Listening to positive statements of participants based on reading the holy book, and spiritual issues in overcoming or felling calm in stressful situations.

Listening to the physical and mental problems, worries, fears, ambivalence sense in early pregnancy and her actions in daily life.

Focus on human creation discuss concerning the status of women in the continuity of creation Talking about the spiritual aspects of the pregnancy and childbearing.

Focus on the concept of trust, resort, patience, kindness.

Blessings of God and the role of it in reinterpreting concept of pregnancy and overcoming the worrisome symptoms of pregnancy.

Book therapy / listening to Qur'an voices for $10 \mathrm{~min}$.

Strengthening individuals' inner hope and powers for coping with pregnancy and childbearing.

Encourages to create a daily spiritual space of time or place at home.

Encouraging to express their feeling after/ during creating a daily spiritual space.

Talking about the experience of participating in religious programs or doing spiritual issues.

Discuss to the effect of spiritual's beliefs on eating habits on the fetus, taking care of oneself in pregnancy.

Encouraging to refer to people who create a positive sense or comfortable with them.

Book therapy / listening to Qur'an voices for $10 \mathrm{~min}$.

Listening to The "Nature's Music" the sound of birds, rivers and waterfalls...

Illustration and slowly moving tone using meditation relaxation technique along or with listening to relaxing music

Discuss the strategy of prayer therapy to reduce the worrisome symptoms of pregnancy related to pregnancy and increase hope

Express the pleasure and responsibility of being a mother from the point of view of the Quran" Divine Responsibility Reward" 
Teaching relaxing muscles with deep breathing for getting rid of the stress. Repeat twice daily for 10 to 15 minutes

Table 2: The comparison of socio-demographic characteristics of participants between two groups

\begin{tabular}{|c|c|c|c|c|c|c|}
\hline \multirow[t]{4}{*}{ Variable } & & \multicolumn{4}{|l|}{ Groups } & \multirow{4}{*}{$\begin{array}{l}\mathrm{P} \\
\text { value }\end{array}$} \\
\hline & & \multirow{2}{*}{\multicolumn{2}{|c|}{ Intervention }} & \multicolumn{2}{|l|}{ Control } & \\
\hline & & & & \multicolumn{2}{|c|}{ Number (percent) } & \\
\hline & & frequency & Percentage & frequency & Percentage & \\
\hline \multirow{4}{*}{$\begin{array}{l}\text { Woman's } \\
\text { Education } \\
\text { woman }\end{array}$} & Guidence & 2 & 6.7 & 2 & 6.7 & \multirow[t]{4}{*}{0.44} \\
\hline & High school & 1 & 3.3 & 1 & 3.3 & \\
\hline & Diploma & 12 & 40 & 10 & 33.3 & \\
\hline & Academic & 15 & 50 & 17 & 56.7 & \\
\hline \multirow{2}{*}{$\begin{array}{l}\text { Woman's } \\
\text { Employment }\end{array}$} & Employed & 14 & 46.7 & 10 & 33.3 & \multirow[t]{2}{*}{0.43} \\
\hline & Housewife & 16 & 53.3 & 20 & 66.7 & \\
\hline \multirow{2}{*}{$\begin{array}{l}\text { Spouses' } \\
\text { employment }\end{array}$} & Employed & 17 & 56.7 & 21 & 70 & \multirow[t]{2}{*}{0.42} \\
\hline & Unemployed & 13 & 43.3 & 9 & 30 & \\
\hline Age (years) & \multirow{2}{*}{$\begin{array}{l}\text { Mean } \pm \\
\text { standard } \\
\text { deviation }\end{array}$} & \multicolumn{2}{|l|}{$25.80 \pm 6.37$} & \multicolumn{2}{|l|}{$24.30 \pm 6.80$} & 0.38 \\
\hline $\begin{array}{l}\text { Gestational age } \\
\text { (week) }\end{array}$ & & \multicolumn{2}{|l|}{$21.80 \pm 1.27$} & \multicolumn{2}{|l|}{$21.60 \pm 1.40$} & $0 . .56$ \\
\hline
\end{tabular}

Table 3: The comparison of quality of life scores between two groups 


\begin{tabular}{|c|c|c|c|c|c|c|}
\hline \multirow[t]{2}{*}{ Variables } & & \multicolumn{2}{|c|}{ Intervention } & \multicolumn{2}{|c|}{ Control } & \multirow[t]{2}{*}{$P$ value } \\
\hline & & Mean & SD & Mean & SD & \\
\hline \multirow[t]{2}{*}{ Physical functioning } & Pre test & 26.75 & 8.43 & 30.25 & 5.14 & 0.05 \\
\hline & Post test & 31.08 & 8.29 & 27.58 & 5.62 & 0.06 \\
\hline$P$ value & Paired t test & 0.0001 & & 0.07 & & \\
\hline \multirow[t]{2}{*}{ Bodily pain } & Pre test & 32.91 & 22.62 & 33.75 & 16.78 & 0.87 \\
\hline & Post test & 33.75 & 25.24 & 38.33 & 20.74 & 0.44 \\
\hline$P$ value & Paired $t$ test & 0.73 & & 0.34 & & \\
\hline \multirow[t]{2}{*}{ Physical role functioning } & Pre test & 6.25 & 5.91 & 5.20 & 5.46 & 0.48 \\
\hline & Post test & 13.33 & 7.65 & 6.25 & 5.44 & 0.0001 \\
\hline$P$ value & Paired $t$ test & 0.0001 & & 0.16 & & \\
\hline \multirow[t]{2}{*}{ Emotional role functioning } & Pre test & 16.66 & 8.47 & 16.66 & 8.47 & 1 \\
\hline & Post test & 29.16 & 9.22 & 16.94 & 8.32 & 0.0001 \\
\hline$P$ value & Paired $t$ test & 0.0001 & & 0.91 & & \\
\hline \multirow[t]{2}{*}{ Social role functioning } & Pre test & 35.41 & 12.74 & 37.08 & 8.97 & 0.56 \\
\hline & Post test & 38.33 & 11.80 & 37.91 & 8.97 & 0.87 \\
\hline$P$ value & Paired t test & 0.18 & & 0.72 & & \\
\hline \multirow[t]{2}{*}{ Mental health } & Pre test & 57.00 & 7.61 & 58.50 & 6.45 & 0.41 \\
\hline & Post test & 62.33 & 8.78 & 57.16 & 6.78 & 0.01 \\
\hline$P$ value & Paired t test & 0.0001 & & 0.45 & & \\
\hline \multirow[t]{2}{*}{ Vitality } & Pre test & 49.79 & 8.44 & 49.16 & 8.64 & 0.77 \\
\hline & Post test & 63.33 & 4.85 & 49.16 & 8.64 & 0.0001 \\
\hline$P$ value & Paired $t$ test & 0.0001 & & 0.18 & & \\
\hline \multirow[t]{2}{*}{ General health perceptions } & Pre test & 35.16 & 7.59 & 33.83 & 5.20 & 0.43 \\
\hline & Post test & 46.66 & 7.46 & 32.33 & 4.09 & 0.0001 \\
\hline$P$ value & Paired $t$ test & 0.0001 & & 0.34 & & \\
\hline \multirow[t]{2}{*}{ Total Quality of Life Score } & Pre test & 85.66 & 5.44 & 86.86 & 3.36 & 0.31 \\
\hline & Post test & 96.46 & 4.44 & 85.76 & 4.04 & 0.0001 \\
\hline$P$ value & Paired $t$ test & 0.0001 & & 0.23 & & \\
\hline
\end{tabular}


Table 4 The linear regression analysis for estimating the risk of poor Quality of Life scores

\begin{tabular}{|lllllll|}
\hline Group & $\mathrm{B}$ & $\mathrm{OR}$ & $\mathrm{R}$ & $\mathrm{R}$ Square & $\mathrm{Cl} 95 \%$ & P value \\
\hline Intervention & -10.70 & -0.78 & 0.78 & 0.62 & $-12.89,-8.50$ & 0.0001 \\
\hline Control & (Ref) & & & & & \\
\hline
\end{tabular}

\section{Figures}

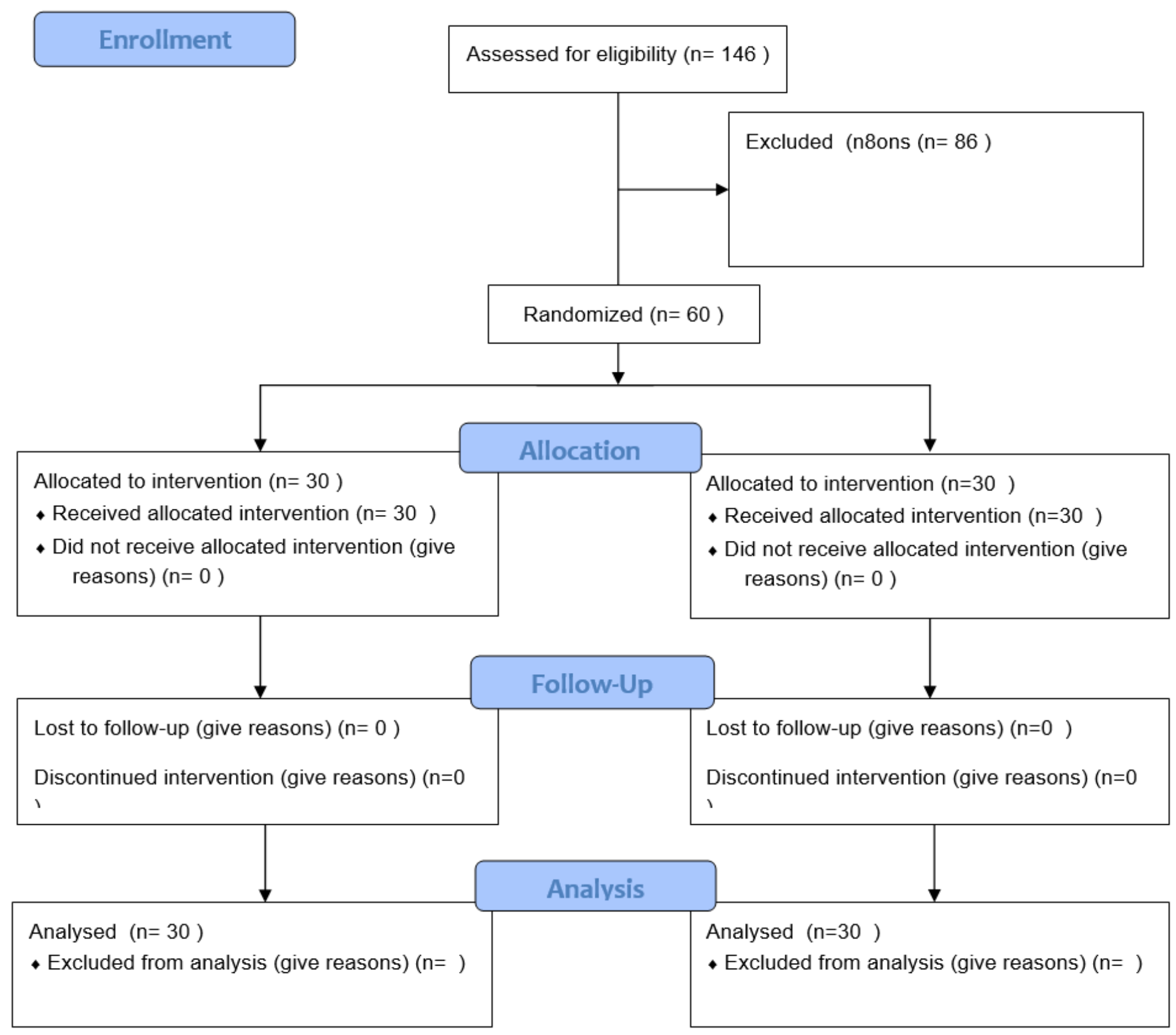


Figure 1

CONSORT 2010 Flow Diagram

\section{Supplementary Files}

This is a list of supplementary files associated with this preprint. Click to download.

- CONSORT2010Checklist.doc

- protocol.......docx 